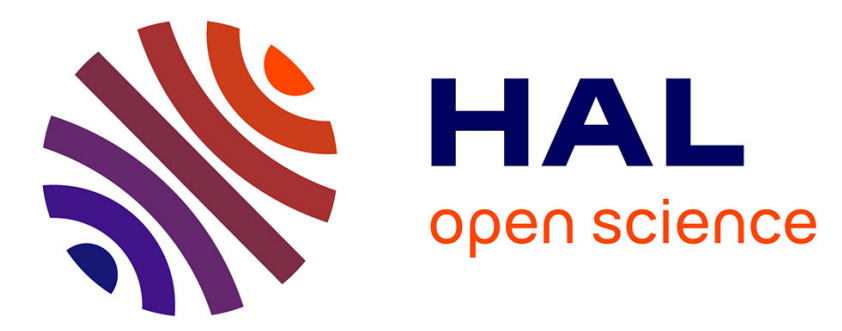

\title{
Malaise dans la finance, malaise dans la mathématisation
} Nicolas Bouleau

\section{To cite this version:}

Nicolas Bouleau. Malaise dans la finance, malaise dans la mathématisation. Revue Esprit, 2009, 352, pp.37-50. halshs-00374588

\section{HAL Id: halshs-00374588 \\ https://shs.hal.science/halshs-00374588}

Submitted on 9 Apr 2009

HAL is a multi-disciplinary open access archive for the deposit and dissemination of scientific research documents, whether they are published or not. The documents may come from teaching and research institutions in France or abroad, or from public or private research centers.
L'archive ouverte pluridisciplinaire HAL, est destinée au dépôt et à la diffusion de documents scientifiques de niveau recherche, publiés ou non, émanant des établissements d'enseignement et de recherche français ou étrangers, des laboratoires publics ou privés. 


\title{
Malaise dans la finance, malaise dans la mathématisation
}

(décembre 2008)

\begin{abstract}
Nicolas Bouleau
De nombreux facteurs ont été avancés pour expliquer la crise des subprimes et l'imprévoyance d'institutions financières pourtant compétentes. Parmi les raisons de fond, on a accusé la titrisation qui mettait sur le marché des produits financiers complexes attachés à des créances douteuses. Plusieurs commentateurs ont également suspecté l'hypermathématisation de la finance de faire illusion de scientificité. Nous reprenons cette question en la poussant plus loin sur le plan philosophique.
\end{abstract}

\section{La crise des subprimes survient alors que la finance est très mathématisée.}

Cette vaste perturbation, dont les dégâts ne sont pas encore circonscrits, est d'une nature spécifique qui révèle la fragilité de certains présupposés économiques : des mises en marché fondées sur des croyances bien établies et cependant contestables. Le rapport de Boissieu du Conseil d'Analyse Economique la situe dans un contexte international de baisse de l'inflation et d'ample liquidité entraînant une diminution sensible de l'aversion au risque et une valorisation concomitante des actifs; elle a été favorisée par de nombreux dysfonctionnements microéconomiques dont évidemment un relâchement des conditions d'attribution des prêts, en particulier sur l'immobilier aux Etats-Unis et déclenchée par des pratiques financières à haut risque autour de la titrisation des créances hypothécaires et du commerce des dérivés de crédit ${ }^{1}$. Le diagnostic porté par George Soros est similaire en mettant davantage l'accent sur la détérioration de la demande intérieure américaine et la baisse du taux d'épargne des ménages, notamment à cause des délocalisations, en comparaison d'une valorisation artificielle de l'immobilier ${ }^{2}$. Le phénomène le plus impressionnant est la propagation de la secousse entraînant des faillites d'établissements et des pertes considérables tant aux Etats-Unis, en Europe qu'au Japon et dans les pays

\footnotetext{
${ }^{1}$ Rapport de Boissieu : P. Artus, J.-P. Betbèze, Ch. de Boissieu, G. Capelle-Blancard et al. La crise des subprimes, La documentation française août 2008.

2 G. Soros La vérité sur la crise des subprimes, Denoël 2008.
} 
émergeant ou exportateurs, induisant des restrictions de crédit aux entreprises au point que les experts ne savent plus si certains secteurs seront épargnés.

En 2006, le prix Nobel Joseph Stiglitz, en ce qui concerne les avances faites aux pays sous-développés, posait la question : "Mais pourquoi les prêteurs sophistiqués, attentifs à maximiser leurs profits, prêtent-ils si souvent trop ?" Et sa réponse, déjà, anticipait celle qui peut être faite à propos des subprimes : "Les prêteurs encouragent l'endettement parce qu'il est rentable. [...] Trop emprunter accroît les risques de crise, et les coûts d'une crise ne sont pas seulement supportés par les prêteurs mais par toute la société"3.

Le rapport de Boissieu pointe très justement la faiblesse des modèles d'estimation de la valeur dans l'incertain utilisés par les banques pour ellesmêmes ou pour leur clients et le caractère abstrait en temps de crise de la méthode du mark to market (qui consiste à évaluer les actifs selon leur cours spot $)^{4}$. Il recommande plus de transparence et d'indépendance des agences de notation, écarte l'argument facile d'incompétence, et plaide pour des normes comptables et une gouvernance internationale au niveau européen et mondial.

De telles mesures sont assurément indispensables, mais elles restent dans l'ambiguiité tant qu'un problème de fond méthodologique n'a pas été explicité qui est la façon d'appréhender les risques dans le monde financier tel qu'organisé aujourd'hui.

A cet égard la crise des subprimes arrive dans un contexte fort différent de celui de la crise de 1929. Les métiers de la finance ne sont plus les mêmes. Il s'est produit en 1973 une rupture épistémologique majeure caractérisée par l'intrusion de mathématiques très avancées dans les savoirs opérationnels des salles des marchés des banques, mathématiques enseignées jusqu'alors au niveau du master 2 ou au delà. Les conséquences de cette "révolution" se firent sentir à différents niveaux ${ }^{5}$.

D'abord par l'instauration de marchés dérivés dans toutes les places financières importantes. En effet les contrats à terme contingents tels que les options $^{6}$ se trouvèrent dès lors gérables de façon commode et se multiplièrent en

\footnotetext{
3 Making Globalization Work, trad. française Un autre monde Fayard 2006 p 363-364.

${ }^{4}$ Le bilan d'une banque est en soi un problème. Les normes comptables IFRS (International Financial Reporting Standards) préconisent le mark to market pour le calcul de la juste valeur (fair value). Le mark to market donne par définition un résultat aussi agité que le sont les cours sur les marchés organisés, il semble plus raisonnable de se référer à un modèle qui tient compte de statistiques temporelles et prend des hypothèses sur les probabilités futures. Mais l'une comme l'autre de ces méthodes ne donneront pas le montant que la banque peut tirer de la vente de ses actifs car elle est en général un trop gros intervenant pour que les cours lui indiquent les prix de vente. Pour la discussion des règles en vigueur cf. P. Amis et S. Sotil-Forgues "Mark to market, mark to model et mark to believe" in Rapport de Boissieu op. cit. p. 163-190.

5 Nous avons tenté une vulgarisation de ces idées fondamentales dans l'ouvrage Martingales et marchés financiers Odile Jacob 1998, et les incompréhensions politiques qui les entourent dans l'article "Les réticences de l'opinion envers la finance" Esprit novembre 1998, 164-176.

${ }^{6}$ Un contrat à terme contigent est une opération qui se déroulera à une échéance future et dont la valeur dépend de l'évolution des cours d'ici l'échéance. Par exemple le droit d'acheter dans trois mois une devise à un prix aujourd'hui fixé, est une option d'achat (appelée call). Le prix d'un tel produit contingent résulte de
} 
rendant aux entreprises des services considérables de protection contre les aléas de fluctuation des cours notamment des devises. Ensuite par une transformation des profils professionnels des praticiens de la finance qui, au lieu d'une formation économique à dominante de sciences humaines, ont été recrutés à la sortie des grandes écoles d'ingénieurs ou des filières mathématiques universitaires. Ceci s'accompagna d'un renouvellement des enseignements accordant une place de choix aux probabilités, à l'analyse stochastique et au calcul différentiel mis au point par le mathématicien japonais Kiyosi Itô. Enfin, ces perfectionnements des marchés financiers et des estimations des contrats à terme eurent également pour conséquence de renforcer le pouvoir de la finance - si l'on peut s'exprimer de façon si générale - en ce sens que les marchés indiquaient mieux que par le passé les anticipations vraisemblables et contribuaient ainsi à influencer les agents y compris les gouvernements, ce qui fit apparaître le passage à la monnaie unique comme une étape indispensable et efficace de la construction européenne.

Après ce changement de paradigme, alors qu'on pensait les outils principaux mis en place, curieusement, ces vingt dernières années la mathématisation s'est poursuivie, essentiellement dans le domaine de la représentation et du calcul des risques pour évaluer l'exposition des établissements bancaires.

Une notion très simple, initiée par la banque JP Morgan au début des années 1990 qui s'est répandue en devenant la méthode la plus utilisée jusqu'à récemment, était fondée sur le principe du seuil. C'est la "value at risk" notée VaR qui représente la perte maximale qu'on a la probabilité $p(=0,95$ ou 0,99$)$ de ne pas dépasser à une échéance donnée 7 . Elle qualifie donc les bornes d'un domaine en dehors duquel on ignore ce qui se passe mais à l'intérieur duquel on reste avec la probabilité $p$. On s'est rendu compte que le critère VaR présentait un défaut. En pratique pour agréger les risques relatifs à des filiales par exemple, ou pour dialoguer entre établissements en vue d'échanges d'actifs risqués, il n'est pas un bon résumé de la situation qu'il quantifie. Il n'est pas difficile de voir qu'il ne permet pas d'estimer le risque sur la réunion de deux portefeuilles connaissant le risque sur chacun d'entre eux ${ }^{8}$.

Pour palier ce défaut, et pour mieux tenir compte de ce qui se passe lorsque le seuil est franchi - question tout de même essentielle qui est passée sous silence par le critère $\mathrm{VaR}$ - on a introduit la taille moyenne des pertes au

\footnotetext{
raisonnements d'une force remarquable mis au point dans les années 1970-80 : le juste prix empêche aussi bien le vendeur que l'acheteur de faire un profit sans risque.

7 Dans le cas (idéal) où l'on connaît parfaitement les lois de probabilités, VaR se calcule aisément : si un portefeuille de $100 €$ a une rentabilité à 10 jours qui suit une loi normale de moyenne $0,4 \%$ et d'écart type $5 \%$, on obtient $\operatorname{VaR}(95 \%)=7,825$ : il y a $95 \%$ de chances que dans 10 jours la perte n'excède pas 7,825€.

8 Par exemple si chacun des deux portefeuilles A et B a une perte de $10 €$ avec probabilité $3 \%$ et nulle avec la probabilité $97 \%$ donc $\operatorname{VaR}(\mathrm{A}, 95 \%)=0 €$ et $\operatorname{Var}(\mathrm{B}, 95 \%)=0 €$, il se peut que pour la réunion des deux portefeuilles la perte soit de $10 €$ avec probabilité $6 \%$ et donc $\operatorname{VaR}(\mathrm{A}+\mathrm{B}, 95 \%)=10 €$. Cf. R. Portait et P. Poncet Finance de marché, Dalloz 2008, p. 933.
} 
delà de VaR qui peut être calculée si on connaît la loi de probabilité de la perte. Plus généralement, on a pu montrer que tout critère de risque vérifiant les règles de bon sens qu'on attend lorsqu'on réunit deux portefeuilles ou lorsqu'on multiplie un portefeuille par un coefficient déterministe, est nécessairement d'une certaine forme mathématique. De tels critères sont appelés mesures cohérentes de risques ${ }^{9}$.

L'intérêt de ce résultat est de souligner l'importance des scénarios. Les lois de probabilités qui régissent les actifs financiers dépendent en général de l'observateur. Les mesures cohérentes de risques suggèrent de se placer sous diverses hypothèses probabilistes pour mener l'analyse des risques et de prendre in fine l'estimation la plus défavorable. On retrouve ici une approche qui était déjà celle des ingénieurs en génie civil. Etant donné que tous les chemins de l'arbre des conséquences qui peuvent mener à la ruine d'un ouvrage (pont, barrage, etc.) ne peuvent être explorés exhaustivement, on envisage a priori certains types de défaillance des éléments de matière et on évalue la probabilité des scénarios conduisant à de tels endommagements. Cette logique des scénarios multiples s'impose de plus en plus dans tous les domaines de la modélisation prospective, y compris bien sûr, dans les études des liens entre les décisions économiques et les modifications du climat.

Ces techniques de quantification des risques ont favorisé la mise des risques en marché que recouvre le vocable de titrisation. Si des compagnies d'assurance, ou des banques, ont à gérer des risques dans leur domaine ou leur région qui sont corrélés, et si ces risques sont indépendants d'une compagnie à une autre, ou moins corrélés, elles ont intérêt à échanger des risques. C'est une idée voisine de la réassurance mais plus générale. Pour instaurer un marché des risques il faut que vendeurs et acheteurs soient capables de les quantifier, d'où l'intérêt de la mathématisation que nous venons d'évoquer. L'idée d'organiser la réassurance en un marché dont les participants sont les compagnies d'assurance, et où le bien échangé est le risque est une idée "géniale" qui fut étudiée déjà par Maurice Allais et Kenneth Arrow dans les années 1950. Elle s'applique aussi bien entre banques pour la gestion de tous leurs risques ${ }^{10}$. Dans le modèle d'Arrow, chaque intervenant a ses propres probabilités subjectives. Ceci s'étend au cas où chacun se préoccupe de plusieurs scénarios (c'est à dire plusieurs lois de probabilité) pour évaluer son risque. Compte tenu du fait qu'ainsi les uns et les autres n'ont pas les mêmes mesures de risque car ils n'interprètent pas les réalités sous-jacentes à chaque actifs de la même façon, que de plus ils n'ont pas les mêmes aversions au risque à cause de leurs fonctions d'utilité propres donc des comportements différents dans l'incertain, des échanges de risque contre paiement peuvent se trouver intéressants pour vendeur et acheteur. Des prix

\footnotetext{
9 Cf. Ph. Artzner, F. Delbaen, J.-M. Eber et D. Health "Thinking coherently" Risk vol. 10 n¹1, nov. 1997.

10 Cf. K. Borch "Equilibrium in a Reinsurance Market" Econometrica vol 30, n³, July 1962; et R. Portait et P. Poncet op. cit. P. 931 et seq.
} 
peuvent être calculés sous les conditions idéales de modélisation compte tenu de l'information à laquelle a accès chaque intervenant.

Seulement, la configuration des produits dérivés de crédits n'incite pas les banques à la prudence. Dans le cas des CDS Credit Default Swap qui sont les plus courants, une banque se défait du risque de contrepartie sur une créance ou un groupe de créances en le transférant à un acheteur de risque contre paiement d'une prime périodique. La banque considère dès lors que son crédit est sans risque et qu'elle n'a plus à obérer de capital contre ce crédit, elle a donc du capital disponible pour proposer de nouveaux crédits. De l'autre côté la banque acheteuse de risque, tant que le défaut éventuel ne s'est pas produit, est rémunérée pour un capital investi nul, ce qui est le nec plus ultra, pourvu que tout aille bien. En revanche si défaut il y a, une somme importante devra être payée par l'acheteur de risque, qui a donc intérêt à ce que les ménages en difficulté de remboursement soient aidés par des relais supplémentaires ${ }^{11}$. On comprend que par la mise en marché, une banque qui va se défaire de son risque de contrepartie a tendance a ne pas attacher trop d'importance à l'évaluation fine des circonstances du prêt qu'elle accorde, et préfère s'en tenir aux appréciations standard des agences de notation.

Pour la gestion des risques complètement probabilisés (montant des dommages éventuels et loi de probabilité connus) l'intérêt d'une mise en marché est certaine. C'est le cas par exemple pour les dommages matériels des accidents de voiture. Mais la plupart des risques ont une composante purement sémantique et interprétative qui repose sur une lecture de l'économie (et qui subsiste dans tous les risques en ce qui concerne l'estimation des queues de lois ${ }^{12}$ ) et, pour de tels risques, faire confiance à l'estimation qu'en fait l'opinion générale - comme on le fait en les mettant sur le marché - est une faute logique, puisque la plupart des intervenants se fondent sur une mathématisation déjà faite tirée des notations des agences et donc n'apportent rien aux insuffisances de compréhension de chacun. Autrement dit, il n'y a de rationalité à l'économie libérale de marché en matière de risque que pour des situations parfaitement calibrées et statistiquement renseignées. La crise des subprimes aura eu cette conséquence de montrer l'erreur d'extrapoler certaines croyances du libre échangisme à des biens abstraits supposant une interprétation de l'avenir ${ }^{13}$. Le marché ne joue pas bien le rôle de sujet interprétant dans les cas qui sont douteux.

\footnotetext{
11 Cf. sur ce point notamment l'analyse de Michel Aglietta La crise, Michalon 2008.

12 Exception faite de quelques phénomènes physiques remarquables, les lois de probabilité sont connues pratiquement par des expériences statistiques qui fournissent des histogrammes. Aussi les très grandes valeurs positives ou négatives qui sont rarement rencontrées ont des probabilités mal évaluées.

13 Cf. P.-N. Giraud Le commerce des promesses, Seuil 2001 et E. Todd Après la démocratie, Gallimard 2008, notamment p. 145 et seq.
} 
Quand la dernière crise est survenue, on a accusé les mathématiques qui rendaient possible cette mise des risques en marché et qui auraient opacifié les raisons des transactions. Il y a quelque chose de juste derrière cette accusation, mais il faut la dégager clairement de toutes sortes d'amalgames.

\section{Les avancées mathématiques récentes sont parfaitement compatibles avec une finance au service d'objectifs sociaux et environnementaux.}

Après le séisme, les mathématiques devinrent le bouc émissaire dans la presse et les média. On n'y comprend plus rien! "Ils" ont perdu tout contact avec l'économie réelle!

Première remarque: il suffit qu'une économie préserve le principe que certains biens sont organisés en marché avec un prix instantané affiché pour que l'idée de la gestion des options par suivi de marché reste pertinente et permette à ces options de rendre des services d'assurance sur les transactions à terme sur ces biens. De même la mise en marché des risques bien calibrés est une bonne idée en soi, elle revient à ne pas mettre tous ses œufs dans le même panier. Accuser les nouvelles méthodes mathématiques de la fragilité de la finance internationale est une erreur, il faut distinguer les quelques idées lumineuses qui sont au cœur de ces méthodes et la quincaillerie, sans doute excessive, qui engendre des abus de confiance.

L'avantage principal du libéralisme économique de tenir le plus grand compte des motivations et des préférences des gens, est préservé si des règles sont imposées pour respecter des limites, éthiques (blanchiment des narcodevises, commerce des organes, etc.), sociales (excessive rémunération du patrimoine et précarité des salariés), et environnementales (ressources vulnérables, forêts, biodiversité, etc.). Trois penseurs économistes sont emblématiques de cette orientation nouvelle: Amartya Sen dont l'œuvre, couronnée du prix Nobel, propose d'aborder l'économie différemment pour ne pas laisser des pays entiers dans la détresse; Joseph Stiglitz, prix Nobel également, qui dénonce le déficit démocratique des instances internationales de régulation ${ }^{14}$ et Nicholas Stern dont le rapport sur le changement climatique montre à l'évidence la myopie de l'économie financière telle qu'organisée aujourd'hui. Les taux d'intérêt actuellement pratiqués avantagent outrageusement le court terme vis à vis du long terme. Ils sont le résultat d'une accumulation de spontanéités irréfléchies sur le mode "un tiens vaut mieux que deux tu l'auras" qui nuisent à l'intérêt général parce que le jeu est à intérêts divergents. Il y a là matière à une prise de responsabilité collective pour un recadrage de la part des banques centrales.

\footnotetext{
14 ainsi que le mode de fonctionnement du FMI et de la Banque mondiale qui, par un cisaillement dû aux aléas des taux de leurs dettes et des changes, aboutit à les paupériser davantage. Cf J. Siglitz op. cit. "le fardeau de la dette" en particulier p. 369 et seq.
} 
Plutôt que de brandir la taxe dite Tobin, solution soi-disant miracle dont les défenseurs n'ont pas mesuré les conséquences sur la recrudescence du gré à gré15, sur la mort des produits dérivés tels que les options (et donc les risques accrus pour les entreprises) ni les difficultés de mise en place d'une instance nécessairement mondiale au pouvoir politique (et de police) considérable, mieux vaut fermer les centres off shore - qui jouent pour la finance le même rôle que les paradis fiscaux pour les rémunérations - et empêchent la progression de la réglementation sous la menace de la fuite des capitaux ${ }^{16}$.

Un royaume infini de liberté absolue, pure et entière, l'Occident s'accroche à ce rêve comme un enfant qui ne veut pas grandir ${ }^{17}$. On peut vivre avec des limites, cela s'organise. Et en ce qui concerne la finance, un peu de mathématiques est indispensable pour cela, donc aussi une vulgarisation bien faite, qui ne laisse pas dans l'ombre les imperfections du système.

\section{Les limites de la mathématisation}

Y aurait-il tout de même quelque part un abus de mathématiques? Nous avons dit qu'il y avait quelques idées lumineuses qui sont adaptables à d'autres politiques économiques et tout un attirail de perfectionnements autour. Est-ce là le point faible? Si l'idée de mettre en marché les contrats risqués est bonne et aisée en économie libérale, d'où vient alors qu'il y ait des crises, des défaillances graves qui se propagent comme on l'a vu avec les prêts hypothécaires américains dits subprimes? Et surtout, comment l'amélioration des modèles et analyses de risque a-t-elle laissé tant d'imprévoyance de la part des établissements bancaires?

Il faut revenir à une analyse plus fondamentale du concept de risque.

C'est la signification de l'événement qui fait le risque. La représentation probabiliste des risques (en probabilités objectives ou en probabilités subjectives $\mathrm{du}$ point de vue de l'agent) est classiquement un couple de grandeurs mathématiques, $1^{\circ}$ une loi de probabilité qui gouverne les états qui peuvent se présenter, $2^{\circ}$ une variable aléatoire, c'est à dire une fonction qui à chaque état fait correspondre un dommage, un coût (compté algébriquement s'il y a aussi des avantages). Par exemple la hauteur d'eau de la Loire à Orléans suit une certaine loi de probabilité, et à chaque hauteur d'eau on peut (théoriquement) assigner un coût des dégâts si inondation il y a.

\footnotetext{
15 Je fais ici l'hypothèse que seules les opérations sur les marchés organisés seraient taxées, au delà on ne voit pas comment l'idée pourrait s'appliquer concrètement.

16 Voir H. Reynier, "La régulation financière internationale et les centres off shore" in Rapport de Boissieu $o p$. cit.p. 259-262.

17 La "philosophie" du livre de George Soros La vérité sur la crise des subprimes, Denoël 2008 en est un exemple typique.
} 
$\mathrm{Au}$ demeurant cette représentation par un couple de grandeurs mathématiques est un schéma de pensée à la fois trop simple et trop idéal pour discuter les risques. Trop idéal parce qu'on n'est quasiment jamais dans une situation où ce modèle est bien renseigné. On ne connaît pas les queues de lois de probabilité parce qu'elles concernent des événements rares sur lesquels les données sont insuffisantes. On ne sait pas quelles corrélations il y a pour évaluer les dommages et on ne dispose pas d'une description complète de ce qui peut se passer. Même dans le cas purement matériel de la force du vent sur les ouvrages d'art, on ignore les ordres de grandeurs des probabilités des phénomènes extrêmes ainsi que les dépendances spatiales et temporelles. Mais surtout le modèle est simpliste parce qu'il évacue les raisons qui nous font nous intéresser aux événements en faisant comme si leur traduction en coût pouvait se faire automatiquement et objectivement.

Le but véritable de l'analyse des risques n'est pas de faire des calculs mais bien d'avancer avec un peu plus de clairvoyance dans l'ordonnancement des faits et des usages sociaux. Il peut s'agir du risque qu'un enfant se fasse écraser en traversant la rue, du risque que l'air de Paris empoisonne ses habitants, que la faillite de telle entreprise en entraîne d'autres, etc.

L'opération intellectuelle qui consiste à probabiliser une situation est fondamentalement une éviction, un effacement $d u$ sens. Elle est, dans le cas particulier de la physique, le résultat du processus de construction des connaissances scientifiques sur les lois de la matière. Mais elle est largement problématique pour tout ce qui concerne le comportement des humains. L'analyse des risques est nécessairement compréhension d'interprétations.

En ce qui concerne les calculs, malgré les précautions méthodologiques, ils ont le tort, inné pourrait-on dire, de dissimuler les ignorances. Parce qu'on ne sait pas quantifier vraiment les risques de contrepartie, ni ceux liés au marché, à la liquidité, encore moins ceux qui seraient dus à une erreur humaine ou à une modification réglementaire, on mélange des calculs très précis et des estimations "à la louche" dont on espère qu'elles n'auront pas de conséquences trop sensibles sur le résultat. Au cours de la fabrication de produits financiers spéciaux comprenant des crédits divers $^{18}$, il y a une perte de signification et une standardisation des qualificatifs de description des situations (réduits le plus souvent aux appréciations des agences de notation) qui éloigne de la réalité. A la limite on a tendance à penser de la même façon un prêt hypothécaire dans l'Iowa ou le Kansas et un crédit à New York sur Madison avenue s'ils sont tous deux bien notés. Comme la théorie mathématique est de loin en avance sur les données, on applique des méthodes sophistiquées à des hypothèses vagues dont on oublie au fur et à mesure des calculs la grossièreté des estimations. Les faillites des entreprises ou des autres banques sont représentées par des

\footnotetext{
18 Titres de créance négociables (TCN), Mortgage backed securities (MBS), Collateralised debt obligations (CDO), etc.
} 
processus poissoniens ${ }^{19}$ dont le paramètre, la fréquence moyenne, est en fait inconnue. C'est une des caractéristiques des mesures dites cohérentes de risque de faire intervenir toute la queue de la loi de probabilité ce qui permet de dire quelque chose sur ce qui se passe au delà du seuil. Mais c'est aussi leur faiblesse car les queues de lois ne sont jamais connues. Donc leur usage entraîne nécessairement le placage d'un modèle mathématique sur une réalité qu'on ignore.

A un second niveau, commercialiser des titres entre établissements dans le cadre naturel de l'économie de marché n'est pas la même chose que de mettre en place de véritables "marchés organisés". Ce point a été clairement révélé par la crise des subprimes. Les transactions de gré à gré, on the counter (otc) en anglais, sont imprégnés de liens symboliques entre les intervenants qui créent des corrélations et augmentent les risques. On façonne des produits "sur mesure" qui sont peu liquides et s'éloignent des conditions d'un marché fondé uniquement sur les prix. Incontestablement c'est une des caractéristiques des relations interbancaires ces dernières années aux Etats-Unis qui explique l'ampleur de la propagation des secousses lors de cette crise.

Enfin, et c'est de loin le plus important, il n'existe aucune mise en commun de moyens d'étude pour procéder à des estimations de risques globaux ou par grands secteurs. C'est ce qui est le plus choquant. Chaque établissement est seul dans le cadre d'une sorte de marché-providence qui équilibrera tous les mouvements si tout va bien et si ce sont les économistes de l'équilibre qui ont raison. Cela devient cynique lorsque après la crise, les grands établissements bancaires et les grands groupes industriels brandissent le risque systémique pour obtenir des aides publiques, alors qu'ils n'ont pas fait la moindre dépense pour l'étudier.

C'est le sens d'un événement qui fait le risque. Prenons un exemple, supposons qu'un type particulier de cancer soit dénombré avec une certaine proportion dans la population française, cette proportion servira d'estimation de ce risque. S'il advient que l'on découvre que la plupart des personnes atteintes par ce cancer avait consommé disons du cannabis vingt ans auparavant, alors tous les consommateurs de cannabis deviennent des malades potentiels, le risque est beaucoup plus important, pourtant seul le sens de l'événement a changé. Ramener un risque à une distribution de probabilités de montants monétaires revient à gommer la majeure part des difficultés. D'abord c'est faire confiance à la mathématisation comme approximation comme s'il s'agissait de la réalité physique alors qu'il est question de signification dont la subjectivité s'infiltre dans toutes les relations sociales des agents. Ce point épistémologique est extrêmement important. La modélisation des risques n'est pas une modélisation comme les autres. On n'est pas dans un processus d'approximation comme

\footnotetext{
19 Dans le cas d'école où les faillites se produiraient "au hasard" et indépendamment les unes des autres, le nombre de faillites dans une année dans une région suivrait une loi de Poisson.
} 
d'habitude en mathématiques appliquées avec les méthodes de discrétisation ou d'éléments finis. Ce sont des interprétations, donc du sens, que l'on transforme en nombre.

La mathématisation s'emploie à prendre en compte des systèmes complexes de risques dans une évaluation par des calculs probabilistes pour les synthétiser par un critère chiffré qui jouera le rôle de première estimation d'un prix. Nous avons vu que des perfectionnements importants ont été apportés récemment à ces procédures notamment avec les mesures dites cohérentes de risques. Mais toutes ces méthodes ont le défaut inné de considérer que le processus interprétatif est clos. Or bien au contraire, loin d'être figé, il est en émergence permanente. Dès qu'une nouvelle lecture est faite, elle engendre des risques nouveaux qui ne sont perçus que par ceux qui la comprennent. Si en 2006, personne ne voit la croissance des prix de l'immobilier et la décroissance de l'épargne des ménages aux Etats-Unis comme un phénomène susceptible de plusieurs interprétations, le risque correspondant n'est pas perçu. La mathématisation des risques évacue ces difficultés dans des hypothèses concernant les queues des lois de probabilité. Il ne suffit pas de dire que cellesci sont mal connues. Elles sont par nature provisoires et fluctuantes en fonction des connaissances interprétatives que les agents dégagent par leur compréhension des phénomènes économiques.

Cette limitation de la mathématisation pertinente vaut aussi bien pour les autres risques qui sont ceux dus aux innovations scientifiques et techniques que pointait Ulrich Beck dès les années 1980. En fait, toute avancée de la connaissance nous fait découvrir des aspects du monde dont nous n'avions pas conscience et engendrent par là même des risques nouveaux. De quelque façon que l'on procède pour les mathématiser on les fige et la mathématique met dans une boîte qui camoufle l'interprétatif. C'est incontournable: la faiblesse des mathématiques c'est qu'elles sont formelles !

\section{Le hasard et le sens}

Avec la notion de risque, on touche une question philosophique profonde à laquelle les modélisateurs semblent peu sensibles et qu'ils escamotent souvent. C'est la marque d'un positivisme plus ou moins inconscient qui ne voit pas malice à appliquer aux affaires humaines les méthodes en usages dans les sciences de la matière.

Le sens et le hasard sont deux catégories qui s'excluent mutuellement comme deux liquides non miscibles. On peut le voir en franchissant l'interface dans l'une ou dans l'autre direction.

Partons d'une situation au hasard. Voici un dessin fait de traits enchevêtrés, disons à la Jackson Pollock, où l'on ne voit rien ni ne comprend rien. Il nous apparaît comme un tirage fortuit parmi d'autres possibles. Mais voici qu'en le contemplant surgit subitement une forme signifiante 
caractéristique, par exemple le visage de Georges Pompidou. Dès lors le dessin n'est plus pour nous au hasard. Il y a du sens. Et c'est irréversible. Plus jamais nous ne pouvons retrouver l'ingénuité dans laquelle nous étions avant d'avoir reconnu cette signification ${ }^{20}$.

Dans l'autre direction, on part d'une situation chargée de sens. C'était le cas en architecture au début du 20ème siècle : comment sortir des "styles" du passé et installer l'art "moderne" ? Comme le réclamait Le Corbusier dans son essai-manifeste Vers une architecture, il fallait abandonner les corniches, les moulures, les décors qui faisaient, depuis la Renaissance, notre lecture du patrimoine bâti. L'architecte Bruno Zevi, théoricien de la modernité apporte une réponse claire. A la question : où placer une fenêtre en dehors des symétries qui sont la marque du classicisme ? il répond "N'importe où ailleurs"21. Le hasard est seul capable d'effacer vraiment le sens. En musique Iannis Xenakis est dans une problématique similaire. Il cherche une harmonie où la succession des sons ne soit plus lue selon la fondamentale, la quinte et par les rapports dynamiques comme la septième de dominante. Il est conduit à utiliser explicitement le hasard pour cela ${ }^{22}$.

La problématique que nous rencontrons en finance avec la calibration des risques pour modéliser mathématiquement par une loi de probabilité et des coûts de dommage est exactement de même nature que l'affaire des mutations au hasard popularisée par Jacques Monod dans Le hasard et la nécessité. Rappelons-en les grandes lignes.

L'idée soulignée par Monod que les mutations de l'ADN se font au hasard et constituent "la roulette de la nature" ne va pas sans difficultés. "Nous disons, écrit-il, que ces altérations sont accidentelles, qu'elles ont lieu au hasard. Et, puisqu'elles constituent la seule source possible de modifications du texte génétique, seul dépositaire à son tour des structures héréditaires de l'organisme, il s'ensuit nécessairement que le hasard est la seule source de toute nouveauté, de toute création dans la biosphère. Le hasard pur, le seul hasard, liberté absolue, mais aveugle, à la racine même du prodigieux édifice de l'évolution"23.

La thèse principale, comme il l'explique dans un style lumineux, est que le hasard opère par un processus à deux niveaux. La "roulette" des mutations propose des patrimoines génétiques nouveaux et, second niveau, l'évolution, par l'action téléonomique de la sélection, trie et garde ce qui a les propriétés requises pour subsister et se reproduire.

\footnotetext{
${ }^{20}$ La force de cette irréversibilité se révèle aussi bien dans les domaines religieux, politique que scientifique : par exemple, Galilée dès lors qu'il reconnaît dans Jupiter et ses satellites un système copernicien, ne peut plus se départir de cette interprétation malgré le fait que, ne possédant à cette époque prénewtonienne que d'une description cinématique des phénomènes, il n'a aucun argument dirimant contre le système géocentrique.

21 B. Zevi Langage moderne de l'architecture Bordas 1981, trad. de Il linguaggio moderno dell'architectura, Einaudi, Turin 1973.

22 Iannis Xenakis, Kéleütha, Ecrits, L'Arche 1994.

${ }^{23}$ Le hasard et la nécessité, Seuil, 1970, p147.
} 
Ce qui est intéressant c'est que la "roulette" de Jacques Monod n'a pas eu que des opposants religieux ou philosophiques. De nombreux scientifiques ont réclamé plus de finesse et de précaution dans l'analyse. Ilya Prigogine considère que la mutation se fait dans le cadre d'une instabilité qui relève de la thermodynamique des systèmes ouverts hors de l'équilibre ${ }^{24}$. Albert Jacquard compare le hasard de Monod à un petit dieu qui explique ce qu'on ne comprend pas comme chez les Grecs ${ }^{25}$.

$\mathrm{Au}$ niveau des mutations également, la critique méticuleuse du statisticien Georges Matheron est que les acides aminés ne se présentent pas statistiquement comme s'ils avaient été tirés au hasard ${ }^{26}$. Il y a, d'après l'expérience, un jeu de la sélection et de l'épigénèse localement et globalement très complexe qui fait qu'on a certaines configurations. Matheron, théoricien disposant d'une large pratique des probabilités et des statistiques dans les domaines les plus divers, va même plus loin et considère qu'on ne peut pas séparer le hasard dû à notre ignorance et celui relatif à la sélection mal connue due au contexte chimique, cellulaire et environnemental. Autrement dit, Matheron accorde qu'il y ait du hasard mais pas n'importe quel hasard et même laisse entendre que dans des circonstances très spécifiées ce puisse être asymptotiquement déterministe. Nous ne savons pas tout. Evidemment, cela pourrait donner de l'eau au moulin du créationnisme et au courant de l' "intelligent design" de certains protestants américains. Mais on sent bien que Matheron y est opposé, au contraire : il veut rendre plus rigoureux, moins emphatique le discours de Monod. La critique de E. Schoffeniels pousse davantage l'hypothèse déterministe en évoquant la physique des brisures d'ADN elle-même ${ }^{27}$.

On voit dans ces critiques de scientifiques, une frustration. Ils n'acceptent pas que la réalité soit si facilement qualifiée d'aléatoire. Le hasard de Monod est ressenti comme un "n'allez pas plus loin, c'est inutile", analogue aux lois positivistes de Comte qui furent considérées comme des limitations de la recherche ${ }^{28}$.

De même, en finance, se satisfaire de la modélisation probabiliste des risques, c'est fermer définitivement la porte à des compréhensions nouvelles.

\footnotetext{
24 I. Prigogine "La thermodynamique et la vie", La Recherche n²4, juin 1972.

25 A. Jacquard "Hasard et génétique des populations" in Le hasard aujourd'hui, op. cit.

26 G. Matheron, Estimer et choisir, Ecole des Mines, 1978 ; Estimating and Choosing, an Essay on Probability and its Practice, trad. Hasofer, Springer 1989.

27 E. Schoffeniels, L'anti-hasard, Gauthier-Villars 1973.

28 Aujourd'hui, les réserves exprimées par Prigogine, Jacquard, Matheron et Schoffeniels se sont avérées chacune porteuse d'un fond de vérité incontestable. Le développement des recherches ces trente dernières années sur les virus et les transferts de gènes ou d'oligonucléotides par vecteurs cationiques lipidiques montre que le contexte chimico-physique des séquences d'ADN est un vaste champ d'investigation dont les savoirs s'organisent en raisonnements et en théories, où l'image de la loterie est dépassée et a perdu l'essentiel de sa valeur de connaissance.
} 
Une modélisation est toujours une interprétation ${ }^{29}$. La plupart des modèles utilisés en météorologie, en hydrologie, pour l'étude du climat, etc., font intervenir le hasard et les probabilités. Là où le cas de la finance est exemplaire et pointe véritablement une limite de la modélisation, en tant que représentation structurée et formalisée, c'est qu'en mathématisant les risques financiers on ambitionne véritablement de modéliser toutes les interprétations possibles d'une situation. Autrement dit, tant qu'il y aura des observateurs qui feront des lectures imprévues des phénomènes économiques, les risques ne se laisseront pas quantifier.

\section{Conclusion}

La mise en marché des risques n'apparaît une bonne idée que pour des risques absolument calibrés, dont la mathématisation (lois, corrélations, etc.) est sans problème. En toute rigueur il ne s'agit que de situations dont l'aléa a une provenance physique, naturelle ou éventuellement sociale si l'interprétation a un rôle mineur (accidents de voitures). Ce ne peut être le cas en finance que dans une situation stationnaire idéale.

A cet égard quel est le rôle des agences de notations ? En attribuant des notes (AAA, AA, BBB, etc.) aux créances titrisées, elles encapsulent le risque, effacent sa nature interprétative, et enclenchent des processus de mathématisation permettant aux banques de vendre et d'acheter sur le marché des risques. Rendre les agences plus indépendantes ne changera rien. Le marché n'est pas un dieu qui règle tous les problèmes. Les risques viennent des craintes et celle-ci résultent de l'intelligence en éveil qui tente de comprendre la complexité du monde économique. Figer avec des notations est naïf, le risque n'est pas "contenu" dans la définition de la créance, il faut laisser la place à l'intelligence future.

Ces remarques montrent que la stabilité systémique ne peut être obtenue sans imposer des règles contraignantes. Nous avons souligné combien il est moralement choquant que les institutions financières ne consacrent globalement qu'une part dérisoire de leurs budgets à étudier et comprendre les risques collectifs interbancaires (bottom-up) de leurs activités de marché.

29 Cf. N. Bouleau Philosophies des mathématiques et de la modélisation, L'Harmattan 1999. 